\title{
Article \\ A Variable Parameter Method Based on Linear Extended State Observer for Position Tracking
}

\author{
Ying Zheng ${ }^{1,2, *}$, Wei Jiang ${ }^{1,2} \mathbb{D}$ and Xinguo Qiu ${ }^{1,2}$ \\ 1 College of Mechanical Engineering, Zhejiang University of Technology, Hangzhou 310023, China; \\ weij@zjut.edu.cn (W.J.); xgqiu@zjut.edu.cn (X.Q.) \\ 2 Key Laboratory of Special Purpose Equipment \& Advanced Processing Technology, Ministry of Education \& \\ Zhejiang Province, Zhejiang University of Technology, Hangzhou 310023, China \\ * Correspondence: greenzy@zjut.edu.cn
}

Citation: Zheng, Y.; Jiang, W.; Qiu, X. A Variable Parameter Method Based on Linear Extended State Observer for Position Tracking. Actuators 2022, 11, 41. https://doi.org/10.3390/ act 110200

Academic Editor: Jinchuan Zheng

Received: 23 November 2021

Accepted: 25 January 2022

Published: 27 January 2022

Publisher's Note: MDPI stays neutral with regard to jurisdictional claims in published maps and institutional affiliations.

Copyright: (c) 2022 by the authors. Licensee MDPI, Basel, Switzerland. This article is an open access article distributed under the terms and conditions of the Creative Commons Attribution (CC BY) license (https:// creativecommons.org/licenses/by/ $4.0 /)$.

\begin{abstract}
This paper presents a control strategy with a linear extended state observer (LESO) and Kalman filter to achieve a high performance of the motion control system. The moment of inertia of the system, which is variable with the robotic joint motion, is estimated in the established model. A LESO with variable gain is designed, which could estimate the states and the total disturbance of the plant without a precision mathematical model. The disturbance caused by variable load and unknown dynamics can be compensated based on the LESO, while the moment of inertia is variable. In order to restrain the process noise and measure the noise of the system, the Kalman filter was applied. Tracking differentiator was utilized to avoid the overshoot of the system for the step signal. The designed control strategy with the LESO and the Kalman filter could improve the tracking performance for the servo system with parametric uncertainties, unknown dynamics, and disturbances. The effectiveness of the proposed method is implemented and validated in the experiment of the robotic joint, for which desired servo tracking performance is achieved with the conditions of load variation and sudden disturbance.
\end{abstract}

Keywords: servomechanism; motion control; linear extended state observer; disturbance compensation; inertia estimation

\section{Introduction}

With the increasing demands for the high performance of motion control systems, advanced control strategies have been applied to the motor drives. For parametric uncertainties, model uncertainties, unknown dynamics, and disturbances, the unknown elements of the dynamics and disturbances need to be estimated and compensated to improve accuracy. During the past several decades, disturbance/uncertainty estimation and attenuation techniques have received considerable attention in mechanical drives [1], including the disturbance observer (DOB) [2,3], unknown input observer (UIO) [4], equivalent input disturbance (EID)-based estimator [5], extended state observer (ESO), etc. [6-10]. The mathematical model of the servo system is not accurate with unknown dynamics and uncertainties, but the disturbance observer methods need a precise model of the plant. Among the previously listed approaches, ESO does not require precise information of the plant; the internal dynamics and external disturbance are treated as total disturbance which could be estimated by ESO and compensated for the plant in real time by an active disturbance rejection controller (ADRC) [11]. ESO is the key part of ADRC, which has been applied in solving a number of industry problems [12-17]. This control strategy draws the information needed to control the plant from ESO, instead of depending on the accurate model of the plant. For system uncertainties, variant load and noise, the disturbance must be estimated and compensated to improve system performance.

The load torque and moment of inertia are variable with the motion of robotic joint; however, the moment of inertia parameter in the nominal model of the servo system is 
constant. In this paper, the moment of inertia and load torque are variable in the model to approach the practical plant in which the total disturbance is estimated and rejected by ADRC to improve system performance. ADRC is an unconventional design strategy, which can actively estimate and compensate for the effects of unknown dynamics and uncertainties in real time. As some of the advantages of ADRC include not requiring a precise model, rejecting disturbance actively, etc., this control method is widely applied in motor servomechanisms [18,19], two-mass actuator systems [20], robotic manipulators [21], etc. Texas Instruments have developed motion control chips based on ADRC technology [22]. The existing research has indicated that the linear extended state observer (LESO) is extremely simple and practical for system control when tuning only one parameter observer bandwidth [23]. LESO has been applied in mechatronic systems with improving the system performance [20].

The Kalman filter (KF) is the minimum-variance linear state estimator for linear dynamic systems with Gaussian noise [24]. In a practical system, there are random noises in sensor measurement and plant running processes. In order to reduce noise, the Kalman filter and various modifications of the Kalman filter are important methods to restrain noise disturbance. The modifications include the extended Kalman filter, the unscented Kalman filter, and the particle filter, which are widely used in target tracking, robotics, navigation, state estimation of the servo system, and others [25]. Since the error of the position sensor can be regarded as Gaussian white noise, the optimal estimation of the speed can be obtained by the Kalman the Filter from a series of position signals with noise [26], so that the Kalman filter could improve system performance by reducing measurement noise.

The objective of this paper was to design a controller based on ADRC and the Kalman filter to improve performance of a servo system of which the moment of inertia is variable. The designed controller was based on variable gain obtained by the estimated inertia and the LESO with the measurement noise reduced by KF. The model was constructed with variable load torque and moment of inertia in real time. Due to the common controller PID causing the large overshoot of output, a tracking differentiator (TD) was used to approximate the system position and speed for reducing output overshoot. This paper is organized as follows. The dynamics of the servomechanism with variable moment of inertia is described in Section 2. In Section 3, the proposed controller based on the LESO and the Kalman filter is presented. The experiment verification is presented in Section 4. Finally, some concluding remarks are given in Section 5.

\section{Dynamic Model of the Servomechanism}

The dynamics of servomechanism is considered as:

$$
\left\{\begin{array}{l}
{\left[\begin{array}{l}
\dot{x}_{1} \\
\dot{x}_{2}
\end{array}\right]=\left[\begin{array}{cc}
0 & 1 \\
0 & -\frac{B_{m}}{J_{m}(t)}
\end{array}\right]\left[\begin{array}{l}
x_{1} \\
x_{2}
\end{array}\right]+\left[\begin{array}{c}
0 \\
\frac{K_{t}}{J_{m}(t) i}
\end{array}\right] u+\left[\begin{array}{c}
0 \\
-\frac{d_{m}(x, t)}{J_{m}(t) i}
\end{array}\right]} \\
y=x_{1}+n(t)
\end{array}\right.
$$

where $x_{1}$ is the angular position of load, $x_{2}$ is the angular velocity of load, $x=\left[x_{1}, x_{2}\right]^{\mathrm{T}}, y$ is the measured position of load, $u$ is the control input, $\mathrm{Bm}$ is the viscous friction coefficient of motor side, $J_{m}(t)$ is the variable moment of inertia of the system, which has been converted to the motor side, $K_{t}$ is the torque coefficient, $i$ is the reduction ratio, $d_{m}(x, t)$ includes the uncertain dynamics and external disturbance viewed as total disturbance converted to motor side, and $n(t)$ is the measurement noise.

$\mathrm{Bm}$ and $K_{t}$ are treated as constant in the model while the moment of inertia $J_{m}(t)$ is variable during the servomechanism motion. Variable feature of $J_{m}(t)$ is considered as similar to the variance of cosine function according to the rotation characteristic of the robotic joint inertia. Load toque is also variable, which is set as a similar cosine function.

As we know, reference signals are often given as a step function causing the control signal step, and making the output largely overshoot. In order to reduce the output overshoot, tracking differentiator is applied to approximate to input position signal and 
velocity. Consider $x_{1 \mathrm{~d}}$ as the input signal of angular position, the tracking differentiator [11] is written as

$$
\left\{\begin{array}{l}
e_{0}=v_{1}-x_{1 \mathrm{~d}} \\
\dot{v}_{1}=v_{2} \\
\dot{v}_{2}=\operatorname{fhan}\left(e_{0}, v_{2}, r_{0}, h\right)
\end{array}\right.
$$

where $v_{1}$ is the output position of the tracking differentiator and $v_{2}$ is its derivative, $e_{0}$ is the tracking error, $h$ is the time step, $r_{0}$ is the parameter related to tracking speed, and function fhan $\left(e_{0}, v_{2}, r_{0}, h\right)$ is

$$
\left\{\begin{array}{l}
d=r_{0} h, d_{0}=h d \\
k=e_{0}+h v_{2}, a_{0}=\sqrt{d^{2}+8 r_{0}|k|} \\
a=\left\{\begin{array}{c}
v_{2}+\frac{a_{0}-d}{2} \operatorname{sign}(k),|k|>d_{0} \\
v_{2}+\frac{k}{h}, \quad|k| \leq d_{0}
\end{array}\right. \\
\text { fhan }=-\left\{\begin{array}{cc}
r_{0} \operatorname{sign}(a), & a>d \\
r_{0} \frac{a}{d}, & a \leq d
\end{array}\right.
\end{array}\right.
$$

Function fhan makes (2) be a time-optimal solution that guarantees fastest convergence from $v_{1}$ to $x_{1 \mathrm{~d}}$ without any overshoot.

The block diagram of the motion control system is illustrated in Figure 1. As shown in the Figure 1, the LESO is the designed observer, KF is the designed Kalman filter used to reduce measurement noise, $x_{1 \mathrm{~d}}$ is the reference signal, $u_{0}$ is the control law without disturbance compensation, $u$ is the control strategy, $z_{3}$ is the estimation of the extended state variable obtained by the LESO, representing the effect of the total disturbance, and $\hat{x}_{1}$ is the angular position with noise suppression by KF. The moment of inertia of the system $J_{m}(t)$ is variable, $v_{1}$ is tracking $x_{1 \mathrm{~d}}$, and $e$ is the error of system.

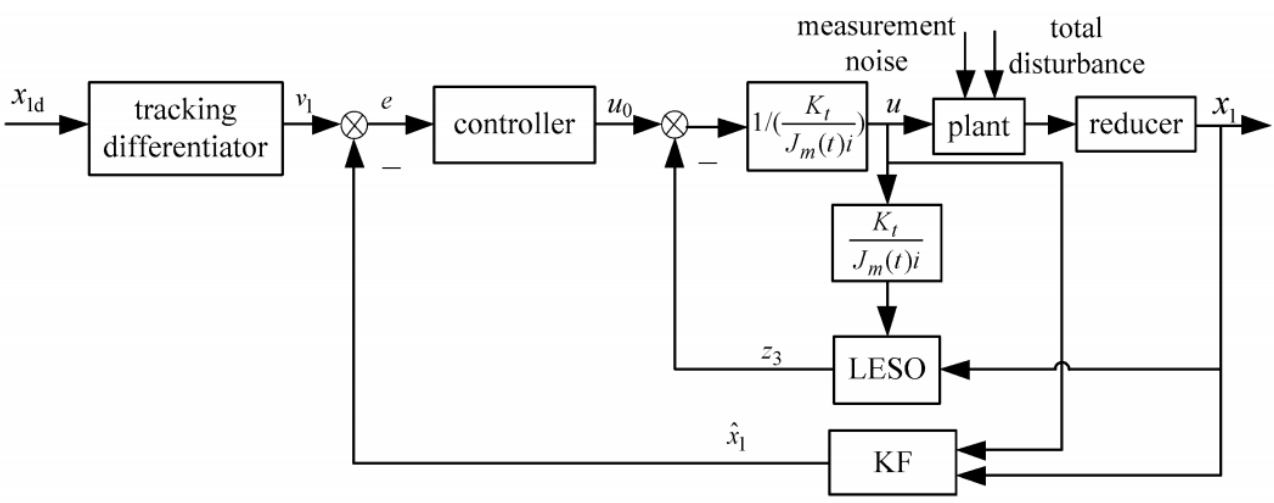

Figure 1. The block diagram of motion control system based on ADRC and KF.

For motion control system-like robots, the moment of inertia $J_{m}(t)$ is not a constant, varying with the robotic joint motion in real time. Consequently, we need to estimate $J_{m}(t)$. As $x_{1}$ is the angular position of load, $x_{2}$ is the angular velocity of load, and $T_{\mathrm{L}}$ is the load torque converted to the motor side; neglecting unknown disturbance, from system (1) we can obtain:

$$
i J_{m} \dot{x}_{2}+i B_{m} x_{2}=K_{\mathrm{t}} u-T_{L}
$$

and define $I_{\tau}=\int_{0}^{t}\left(K_{t} u-T_{L}\right) d t$.

From (4), we obtain:

$$
\left\{\begin{array}{l}
i J_{m} x_{2}+i B_{m} x_{1}=I_{\tau} \\
I_{\tau}(k+1)=I_{\tau}(k)+\left[K_{t} u(k+1)-T_{L}(k+1)\right] T
\end{array}\right.
$$

where $T$ is the sample time, $k=0,1,2 \ldots$

Define $\boldsymbol{\alpha}=\left[i J_{m} i B_{m}\right]^{\mathrm{T}}, \boldsymbol{f}=\left[\begin{array}{ll}x_{2} & x_{1}\end{array}\right]$, substituting into (5), and its discrete form is:

$$
I_{\tau}(k)=f(k) \boldsymbol{\alpha}
$$


The estimation error is:

$$
\varepsilon(k)=I_{\tau}(k)-f(k) \hat{\boldsymbol{\alpha}}
$$

where $\hat{\alpha}$ is the estimation of $\alpha$.

Define $\boldsymbol{I}_{\tau}=\left[I_{\tau}(1), I_{\tau}(2), \cdots I_{\tau}(m)\right]^{\mathrm{T}}, \boldsymbol{F}=[\boldsymbol{f}(1), \boldsymbol{f}(2), \cdots \boldsymbol{f}(m)]^{\mathrm{T}}$.

where $m$ is the amount of sample period.

The error evaluation function $J(\hat{\boldsymbol{\alpha}})$ is:

$$
J(\hat{\boldsymbol{\alpha}})=\left(\boldsymbol{I}_{\tau}-\boldsymbol{F} \hat{\boldsymbol{\alpha}}\right)^{\mathrm{T}} \boldsymbol{W}_{m}\left(\boldsymbol{I}_{\tau}-\boldsymbol{F} \hat{\boldsymbol{\alpha}}\right)
$$

where $\boldsymbol{W}_{m}$ is the weight matrix, $\boldsymbol{W}_{m}=\operatorname{diag}\left(w_{1}, w_{2}, \cdots, w_{m}\right), w_{i} \in(0,1], i=1,2, \ldots m$, and $w_{i}$ is the confidence weight of testing value in $i$ th order.

Minimum of $J(\hat{\boldsymbol{\alpha}})$ is:

$$
\begin{aligned}
& \left.\frac{\partial J(\hat{\boldsymbol{\alpha}})}{\partial \alpha}\right|_{\substack{\alpha=\hat{\alpha}\\
}}=-2 \boldsymbol{F}^{\mathrm{T}} \boldsymbol{W}_{m}\left(\boldsymbol{I}_{\tau}-F \hat{\boldsymbol{\alpha}}\right)=0 \\
& \Rightarrow \hat{\boldsymbol{\alpha}}=\left(\boldsymbol{F}^{\mathrm{T}} \boldsymbol{W}_{m} \boldsymbol{F}\right)-1 \boldsymbol{F}^{\mathrm{T}} \boldsymbol{W}_{m} \boldsymbol{I}_{\tau}
\end{aligned}
$$

The recursive least square method is:

$$
\begin{gathered}
\hat{\boldsymbol{\alpha}}(m+1)=\hat{\boldsymbol{\alpha}}(m)+\boldsymbol{K}(m+1)\left[I_{\tau}(m+1)-\boldsymbol{f}(m+1) \hat{\boldsymbol{\alpha}}(m)\right] \\
\boldsymbol{P}(m+1)=\boldsymbol{P}(m)-\boldsymbol{K}(m+1) \boldsymbol{f}(m+1) \boldsymbol{P}(m) \\
\boldsymbol{K}(m+1)=\boldsymbol{P}(m) \boldsymbol{f}^{\mathrm{T}}(m+1)\left[w_{m+1}^{-1}+\boldsymbol{f}(m+1) \boldsymbol{P}(m) \boldsymbol{f}^{\mathrm{T}}(m+1)\right]^{-1}
\end{gathered}
$$

where $\hat{\boldsymbol{\alpha}}(m)$ is the estimation value at time $m T ; I_{\tau}(m+1)$ is the testing value at time $(m+1) T$, which could be obtained by $(5) ; I_{\tau}(m+1)-\boldsymbol{f}(m+1) \hat{\boldsymbol{\alpha}}(m)$ is the estimation error; $\boldsymbol{K}(m+1)$ is the gain matrix update; $\boldsymbol{P}(m)=\left[\boldsymbol{F}^{T} \boldsymbol{W}_{m} \boldsymbol{F}\right]^{-1} ; w_{m+1}$ is the $(m+1)$ th order weight of $\boldsymbol{W}_{m}, m=0,1,2 \ldots$

So, we can obtain $\hat{J}_{m}(t)$ which is the estimation value of $J_{m}(t)$ from (10), for $J_{m}(t)$ is varying with the robotic joint motion.

\section{Controller Design Based on LESO and KF}

\subsection{Kalman Filter}

Considering the process noise and measurement noise, and in order to obtain precise state values of the system, the Kalman filter is applied to filter the system noise. System (1) can be written as:

$$
\begin{gathered}
\dot{x}=A_{+} \boldsymbol{x}+\boldsymbol{B}_{+} u+E h^{\prime} \\
y=C x+n(t)
\end{gathered}
$$

where $\boldsymbol{A}_{+}=\left[\begin{array}{cc}0 & 1 \\ 0 & -\frac{B_{m}}{J_{m}(t)}\end{array}\right], \boldsymbol{B}_{+}=\left[\begin{array}{c}0 \\ \frac{K_{t}}{J_{m}(t) i}\end{array}\right], \boldsymbol{E}=\left[\begin{array}{l}0 \\ 1\end{array}\right], \boldsymbol{C}=\left[\begin{array}{ll}1 & 0\end{array}\right], x=\left[x_{1}, x_{2}\right]^{\mathrm{T}}$, $h^{\prime}=-\frac{d_{m}(x, t)}{J_{m}(t) i}$.

System (11) is written as following discrete form:

$$
\left\{\begin{array}{l}
x_{k+1}=x_{k}+T\left(A_{+} x_{k}+B_{+} u_{k}\right)=\left(I+A_{+} T\right) x_{k}+B_{+} T u_{k} \\
y_{k}=C x_{k}
\end{array}\right.
$$

where $x_{k}, x_{k+1}$ is the state vector at time $t_{k}$, and $t_{k+1}$, respectively, $u_{k}$ is the input control variable at time $t_{k}, I$ is the identity matrix, and $y_{k}$ is the measurement.

Define $\boldsymbol{\Phi}=\boldsymbol{I}+\boldsymbol{A}_{+} T, \boldsymbol{G}=\boldsymbol{B}_{+} T$, the discrete equation of the system (11) is:

$$
\left\{\begin{array}{l}
x_{k+1}=\boldsymbol{\Phi} x_{k}+G u_{k}+w_{k} \\
y_{k}=C x_{k}+v_{k}
\end{array}\right.
$$


where $w_{k}$ is the system noise with covariance $Q, v_{k}$ is measurement noise with covariance $\boldsymbol{R}$, and the Kalman filter equations are given as:

$$
\begin{gathered}
\hat{\boldsymbol{x}}_{k+1 / k}=\boldsymbol{\Phi} \hat{\boldsymbol{x}}_{k}+\boldsymbol{G} u_{k} \\
\boldsymbol{P}_{k+1 / k}=\boldsymbol{\Phi} \boldsymbol{P}_{k} \boldsymbol{\Phi}^{T}+\boldsymbol{Q} \\
\boldsymbol{K}_{k+1}=\boldsymbol{P}_{k+1 / k} \boldsymbol{C}^{T}\left(\boldsymbol{C} \boldsymbol{P}_{k+1 / k} \boldsymbol{C}^{T}+\boldsymbol{R}\right)^{-1} \\
\boldsymbol{P}_{k+1}=\left(\boldsymbol{I}-\boldsymbol{K}_{k+1} \boldsymbol{C}\right) \boldsymbol{P}_{k+1 / k} \\
\hat{\boldsymbol{x}}_{k+1}=\hat{\boldsymbol{x}}_{k+1 / k}+\boldsymbol{K}_{k+1}\left(y_{k}-\boldsymbol{C} \hat{\boldsymbol{x}}_{k+1 / k}\right)
\end{gathered}
$$

for $k=1,2, \ldots$, where $\hat{x}_{k+1 / k}$ is the a priori estimate of the state $x_{k}, \hat{x}_{k+1}$ is the a posteriori estimate of the state $x_{k}, K_{k+1}$ is the Kalman gain, $\boldsymbol{P}_{k+1 / k}$ is the covariance of the a priori estimation error $\boldsymbol{x}_{k+1}-\hat{\boldsymbol{x}}_{k+1 / k}$, and $\boldsymbol{P}_{k+1}$ is the covariance of posteriori estimation error $x_{k+1}-\hat{x}_{k+1}$. The Kalman filter is initialized with:

$$
\begin{gathered}
\hat{\boldsymbol{x}}_{0}=\mathrm{E}\left(\boldsymbol{x}_{0}\right) \\
\boldsymbol{P}_{0}=\mathrm{E}\left[\left(\boldsymbol{x}_{0}-\hat{x}_{0}\right)\left(x_{0}-\hat{x}_{0}\right)^{\mathrm{T}}\right]
\end{gathered}
$$

where $\mathrm{E}(\cdot)$ is the expectation operator.

\subsection{LESO Design}

The linear extended state observer (LESO) is widely used to estimate states of the system and the extended state could represent the total disturbance [23]. The extended state form of the motion control system (1) is:

$$
\left\{\begin{array}{l}
\dot{x}_{1}=x_{2} \\
\dot{x}_{2}=x_{3}+\frac{K_{t}}{J_{m}(t) i} u \\
\dot{x}_{3}=h(x, t) \\
y=x_{1}+n(t)
\end{array}\right.
$$

where $x_{3}=-\frac{d_{m}(x, t)}{J_{m}(t) i}-\frac{B_{m}}{J_{m}(t) i} x_{2}$ is the extended state, which is also the effect of the total disturbance, and $x_{3}$ is differentiable.

The LESO of the system (21) is designed as:

$$
\left\{\begin{array}{l}
\dot{z}_{1}=z_{2}+l_{1}\left(x_{1}-z_{1}\right) \\
\dot{z}_{2}=z_{3}+l_{2}\left(x_{1}-z_{1}\right)+\frac{K_{t}}{\hat{J}_{m}(t) i} u \\
\dot{z}_{3}=l_{3}\left(x_{1}-z_{1}\right)
\end{array}\right.
$$

where $z=\left[z_{1}, z_{2}, z_{3}\right]^{\mathrm{T}}$ is the estimation of the system extended states $x=\left[x_{1}, x_{2}, x_{3}\right]^{\mathrm{T}}$, which is obtained by LESO, and $l_{i}, i=1,2,3$, are the observer gain parameters to be designed.

The characteristic polynomial of (22) is $s^{3}+l_{1} s^{2}+l_{2} s+l_{3}, l_{i}, i=1,2,3$, are selected to make (22) be Hurwitz. For simplicity, let:

$$
s^{3}+l_{1} s^{2}+l_{2} s+l_{3}=\left(s+\omega_{0}\right)^{3}=s^{3}+3 \omega_{0} s^{2}+3 \omega_{o}^{2} s+\omega_{o}^{3}
$$

From (23), we obtain $l_{1}=3 \omega_{0}, l_{2}=3 \omega_{0}^{2}, l_{3}=\omega_{0}^{3}$. Here, $\omega_{0}$ is the observer bandwidth, which is the only tuning parameter of LESO, $\omega_{0}>0$.

Theorem 1. Assuming $h(x, t)$ is bounded, there exists $M>0, \omega_{0}>0$ such that $\left|\widetilde{x}_{i}\right|=$ $\left|x_{i}-z_{i}\right| \leq M, i=1,2,3$. 
Proof. Since $h(x, t)$ is bounded, there exists positive constant D such that $|h(\boldsymbol{x}, t)| \leq D$. Define estimation error $\widetilde{x}_{i}=x_{i}-z_{i}, i=1,2,3$. From (21) and (22), the observer estimation error is:

$$
\left\{\begin{array}{l}
\dot{\widetilde{x}}_{1}=\widetilde{x}_{2}-3 \omega_{0} \widetilde{x}_{1} \\
\dot{\tilde{x}}_{2}=\widetilde{x}_{3}-3 \omega_{0}^{2} \widetilde{x}_{1} \\
\dot{\tilde{x}}_{3}=h(x, t)-\omega_{0}^{3} \widetilde{x}_{1}
\end{array}\right.
$$

(24) can be written as:

$$
\dot{\tilde{x}}=A \widetilde{x}+B h(x, t)
$$

where $\widetilde{x}=\left[\widetilde{x}_{1}, \widetilde{x}_{2}, \widetilde{x}_{3}\right], A=\left[\begin{array}{ccc}-3 \omega_{0} & 1 & 0 \\ -3 \omega_{0}^{2} & 0 & 1 \\ -\omega_{0}^{3} & 0 & 0\end{array}\right], \boldsymbol{B}=\left[\begin{array}{l}0 \\ 0 \\ 1\end{array}\right]$.

Solving (25), we can obtain:

$$
\widetilde{\boldsymbol{x}}=\exp (A t) \widetilde{\boldsymbol{x}}(0)+\int_{0}^{t} \exp (A(t-\tau) B h(x, t) d \tau
$$

Note that:

$$
\|\widetilde{x}\|_{\infty}=\left\|\exp \left({ }_{A} t\right) \widetilde{x}(0)\right\|_{\infty}+\left\|\int_{0}^{t} \exp \left(A_{A}(t-\tau)\right)_{B} h(x, t) d \tau\right\|_{\infty} \leq\left\|\exp \left({ }_{A} t\right)\right\|_{\infty}\|\widetilde{x}(0)\|_{\infty}+D \int_{0}^{t}\left\|\exp \left({ }_{A}(t-\tau)\right)\right\|_{\infty}\|B\|_{\infty} d \tau .
$$

From (25), matrix $A$ has three same eigenvalues $-\omega_{0}$, then there exists constant $K>0$, such that:

$$
\begin{gathered}
\|\exp (A t)\|_{\infty} \leq K \exp \left(-\omega_{0} t\right), t>0 . \\
\|\exp (A(t-\tau))\|_{\infty} \leq K \exp \left(-\omega_{0}(t-\tau)\right), t>\tau .
\end{gathered}
$$

Combining (27), (28) and (29) yields:

$$
\|\widetilde{\boldsymbol{x}}\|_{\infty} \leq K \exp \left(-\omega_{0} t\right)\|\widetilde{\boldsymbol{x}}(0)\|_{\infty}+\frac{K D}{\omega_{0}}\left(1-\exp \left(-\omega_{0} t\right)\right) \leq K\|\widetilde{\boldsymbol{x}}(0)\|_{\infty}+\frac{K D}{\omega_{0}}=M, M>0 .
$$

So, there exists constant $M>0$, such that $\left|\widetilde{x}_{i}\right|=\left|x_{i}-z_{i}\right| \leq M, i=1,2,3$.

From (30), we can obtain that the larger the observer bandwidth $\omega_{0}$ is, the more accurate the states estimation will be. If $\omega_{0}$ is chosen as the suitable value, $z_{i}$ will approximate to $x_{i}, i=1,2,3$.

\subsection{Controller Design}

Due to the large overshoot of the system output when the input signal is the step function, a tracking differentiator is used to reduce the overshoot, as mentioned before. The system error is the tracking differentiator output $v_{1}$, subtracting the system angular position.

The control law is designed as:

$$
u=\frac{u_{0}-z_{3}}{\frac{K_{t}}{\hat{J}_{m}(t) i}}=\frac{\hat{J}_{m}(t) i\left(u_{0}-z_{3}\right)}{K_{t}} .
$$

Substituting (31) into (21), the system is described as a cascade integral form:

$$
\left\{\begin{array}{l}
\dot{x}_{1}=x_{2} \\
\dot{x}_{2}=u_{0} \\
y=x_{1}+n(t)
\end{array}\right.
$$

where $u_{0}$ is designed as the linear controller. 
Define $\hat{x}_{1}$ as the position estimation of $y$ by the Kalman filter, i.e., system output reducing the measurement noise by filtering.

Let:

$$
u_{0}=k_{p}\left(v_{1}-\hat{x}_{1}\right)+k_{d}\left(v_{2}-\hat{x}_{2}\right)+\dot{v}_{2}
$$

where $k_{p}$ and $k_{d}$ are the controller gain parameters.

Define $e=v_{1}-\hat{x}_{1}$.

Combining (33) with (32) yields:

$$
k_{p} e+k_{d} \dot{e}+\ddot{e}=0 .
$$

$k_{p}$ and $k_{d}$ are selected to make $s^{2}+k_{d} s+k_{p}$ Hurwitz, then $e \rightarrow 0$. For simplicity, let:

$$
s^{2}+k_{d} s+k_{p}=\left(s+\omega_{c}\right)^{2}=s^{2}+2 \omega_{c} s+\omega_{c}^{2}
$$

so $k_{p}=\omega_{c}^{2}, k_{d}=2 \omega_{c}$, where $\omega_{c}$ is the controller bandwidth, which is the only tuning parameter of the controller. $\omega_{c}$ should be adjusted based on the requirements of performance and stability margin.

Substituting (33) into (31), ADRC control law is given by:

$$
u=\frac{\hat{J}_{m}(t) i\left(k_{p} e+k_{d} \dot{e}+\dot{v}_{2}-z_{3}\right)}{K_{t}}
$$

\section{Experiment Verification}

In this section, the robotic joint servo system is investigated to verify the effectiveness of the proposed control strategy. We developed an experimental platform with variable load, which consisted of a slider and a whirling arm. The inertia of the load varied when the slider moved, changing the distance between the slider and the center of the whirling arm. The verification platform for the motion control system was set up in Figure 2. The gyroscope sensor was used to measure the actual position of the whirling arm with the slider. The torque sensor was applied to measure load torque, which was used to estimate the inertia of the load. The permanent magnet synchronous motor (PMSM) was selected as the actuator. The DSP-TMS320F28335 were the core part to control the servomechanism with control strategies. DSP-TMS320F28335 was used to produce the control law, which was the position controller output to track the position signals.

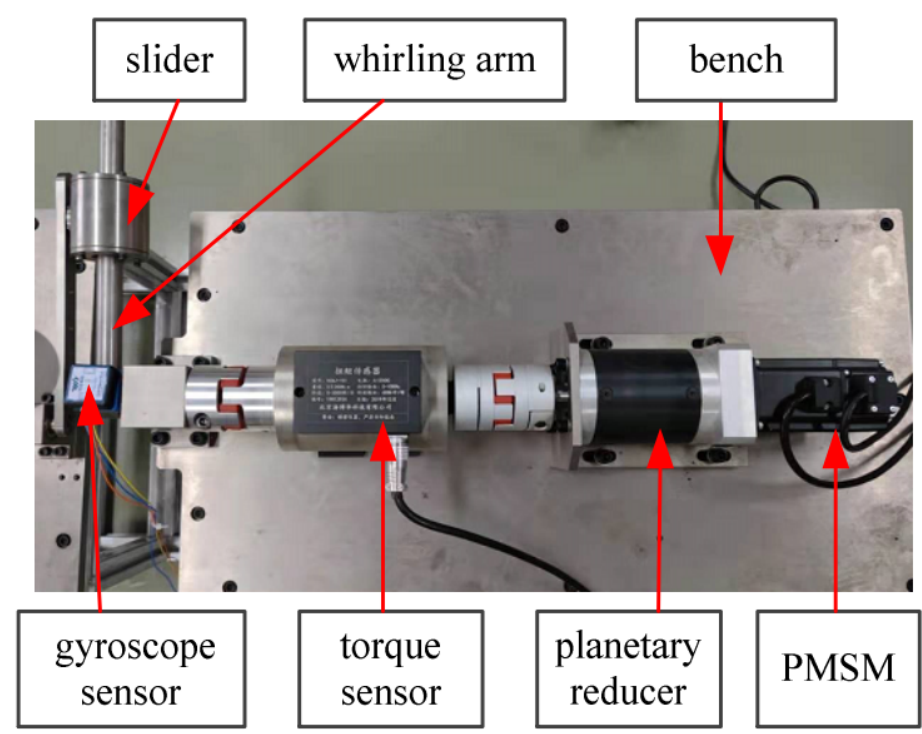

Figure 2. Experimental platform of servomechanism with variable load. 
In the experiments, we take the following two controllers to compare:

(1) C1: The proposed controller based on LESO and estimated inertia with KF in this paper. The control parameters were set as: $k_{p}=25, k_{d}=10, \omega_{0}=100$. Tracking differentiator parameters were given as: $h=0.001, r_{0}=15$. Kalman filter parameters were: $\mathbf{Q}=\operatorname{diag}(0.05,0.05), \mathbf{R}=0.01, \mathbf{P}_{0}=\operatorname{diag}(0.1,0.1)$. The parameter of the inertia estimation was set as: $\mathbf{W}_{10}=\operatorname{diag}(0.2,0.2, \cdots, 0.2)$.

(2) C2: PID controller was used in C2 with the controller parameters $k_{p}=30, k_{i}=20$, $k_{d}=20$.

The parameters of the experimental platform are shown in Table 1.

Table 1. Parameters of the experimental platform.

\begin{tabular}{cc}
\hline Parameters & Value \\
\hline Rated speed $n$ & $3000 \mathrm{rpm}$ \\
Torque coefficient $K_{t}$ & $0.112 \mathrm{~N} \cdot \mathrm{m} / \mathrm{A}$ \\
Motor Inertia $J$ & $1.75 \times 10^{-5} \mathrm{~kg} \cdot \mathrm{m}^{2}$ \\
Reduction ratio $i$ & $100: 1$ \\
\hline
\end{tabular}

In the first case, the sinusoidal-like input signal $x_{1 \mathrm{~d}}(t)=60-60 \sin (0.3 \pi t+\pi / 2)^{\circ}$ was utilized. In order to analyze the tracking performance of the two controllers, the maximum, average, and standard deviation of the tracking errors marked as $M, \mu$ and $\sigma$ were utilized.

The experimental results are presented in Figures 3-6. Specifically, as shown in Figure 3, the actual position tracked the input signal well with the proposed controller $\mathrm{C} 1$, and $\mathrm{C} 1$ had the better performance compared with $\mathrm{C} 2$. The position tracking errors of the two controllers in Figure 4 illustrate that the tracking error of $C 1$ was smaller than that of $\mathrm{C} 2$, which means $\mathrm{C} 1$ had the better tracking performance than $\mathrm{C} 2$. The performance indices are exhibited in Table 2, demonstrating that C1 can achieve higher tracking accuracy than C2. The states estimation by LESO is shown in Figure 5. As seen, $x_{1}$ and $x_{2}$ can be well tracked by $\hat{x}_{1}$ and $\hat{x}_{2}$, which were estimated by the LESO. The tracking errors of $x_{1}$ and $x_{2}$ tended to zero quickly, which means the LESO had good convergence rate and stability. The RMSE of $x_{1}$ estimation error and $x_{2}$ estimation error were $0.00901^{\circ}$ and $1.087^{\circ}$, respectively. The system uncertainties and disturbances were compensated by the LESO, so that C1 had a better control performance than that of $\mathrm{C} 2$, with a relative poor robustness against the unknown dynamics. Control input voltage of the proposed controller is exhibited in Figure 6. As shown, it was bounded.

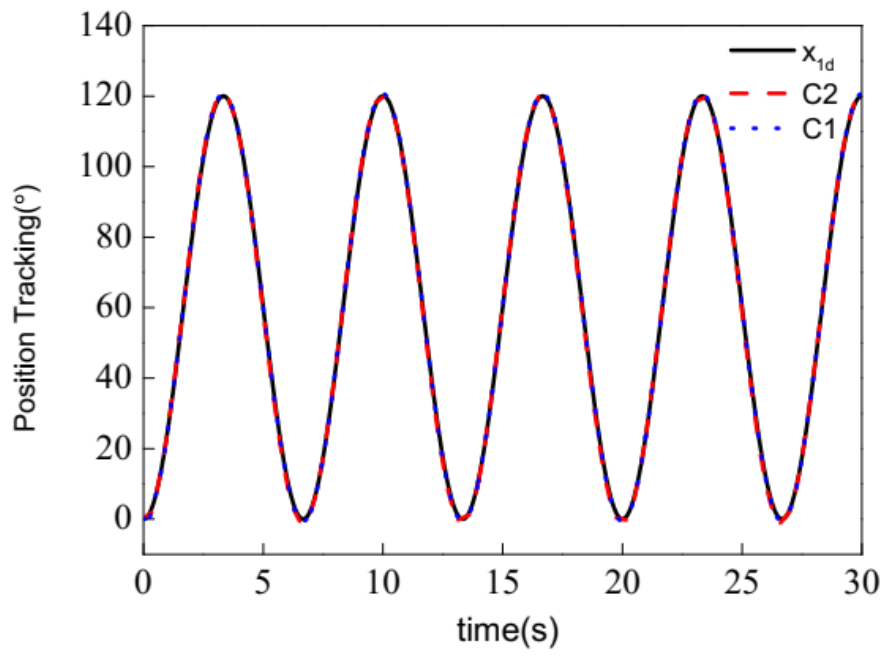

Figure 3. Position tracking performance of $\mathrm{C} 1$ and $\mathrm{C} 2$. 


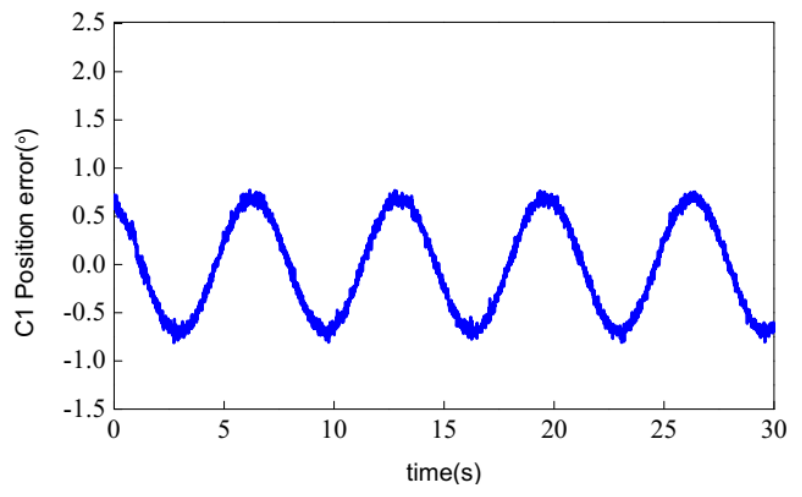

(a)

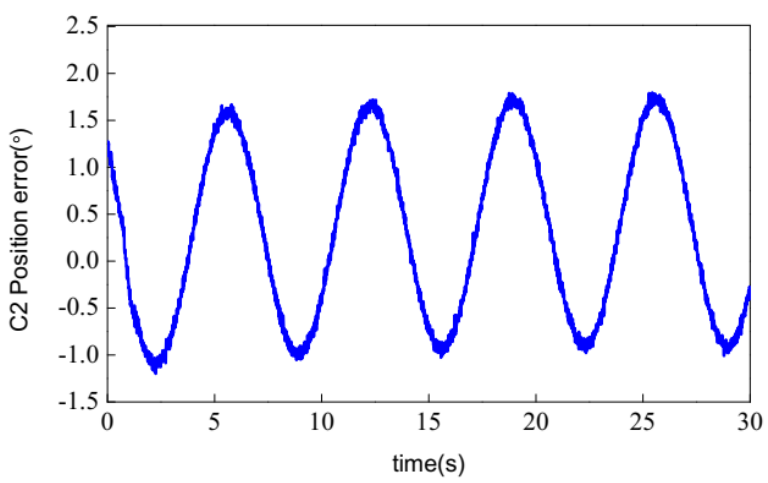

(b)

Figure 4. Position tracking errors of $\mathrm{C} 1$ and $\mathrm{C} 2$.

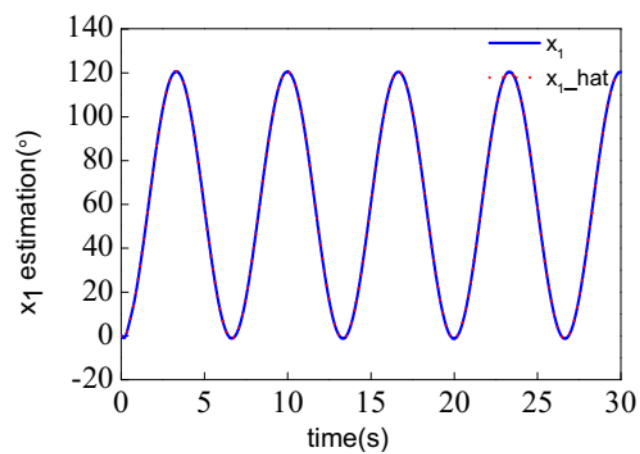

(a)

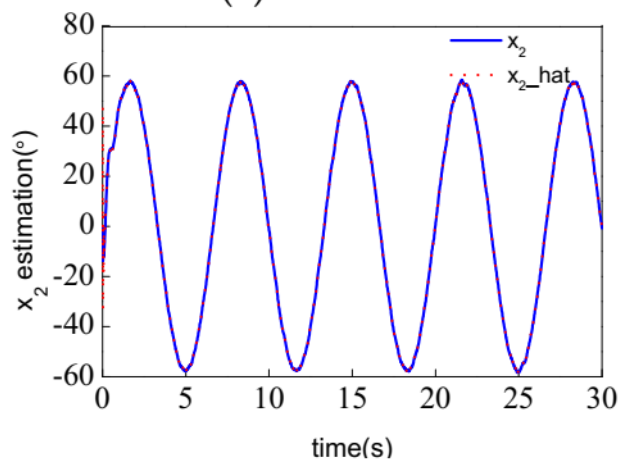

(c)

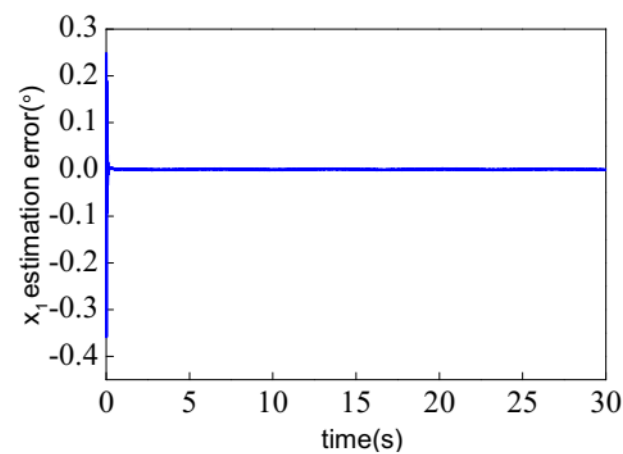

(b)

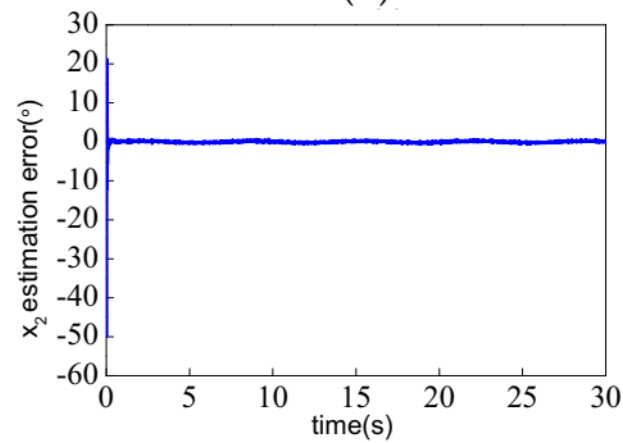

(d)

Figure 5. States estimation and estimation error of $x_{1}$ and $x_{2}$.

In order to further verify the control performance of the proposed strategy, the input signal $x_{1 \mathrm{~d}}$, which was the step signal with maximum angle $120^{\circ}$, is given in Figure 7 . For this motion trajectory, the setpoint jump was not appropriate for most dynamic systems making a step. In order to avoid these undesired characteristics, TD was used to make the output of the plant reasonably follow the input signal. The position tracking of the two controllers is illustrated in Figure 8. As shown, the setpoint jump of the signal caused overshoot of the tracking signal with the PID controller C2. The proposed controller was applied to avoid the overshoot by using TD, and we obtained that $\mathrm{C} 1$ better guaranteed tracking performance in the transient process than $\mathrm{C} 2$. The settling time of the system with $\mathrm{C} 1$ was $0.63 \mathrm{~s}$, whereas the steady-state error was $0.044^{\circ}$. The tracking error of the two controllers are shown in Figure 9. Figure 9 shows that the tracking error of the proposed 
controller $\mathrm{C} 1$ was much smaller than that of $\mathrm{C} 2$ in the regulation stage, although the tracking error was large at the step time.

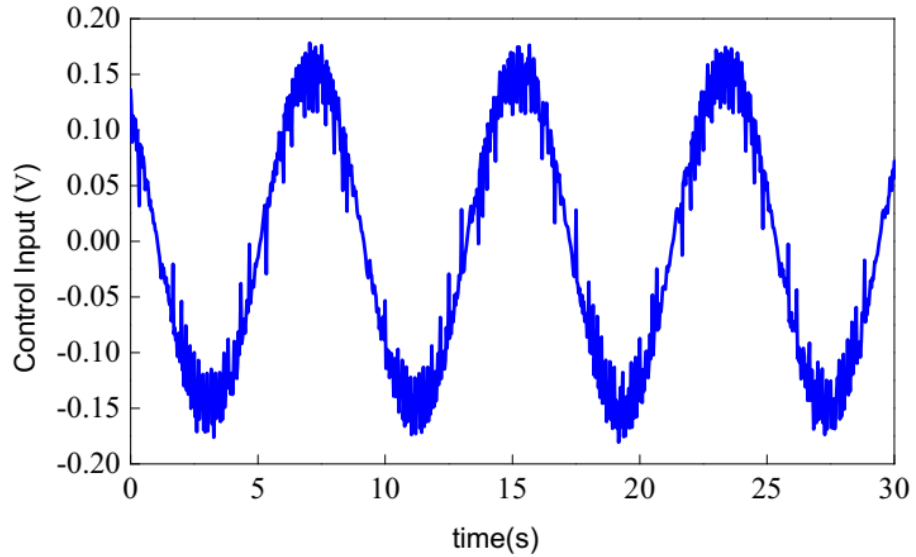

Figure 6. Control input voltage of $\mathrm{C} 1$.

Table 2. Performance indices of the two controllers.

\begin{tabular}{cccc}
\hline Indices $\left(^{\circ}\right)$ & $\boldsymbol{M}$ & $\boldsymbol{\mu}$ & $\boldsymbol{\sigma}$ \\
\hline $\mathrm{C} 1$ & 0.7742 & 0.0212 & 0.4929 \\
$\mathrm{C} 2$ & 1.7925 & 0.2693 & 0.9348 \\
\hline
\end{tabular}

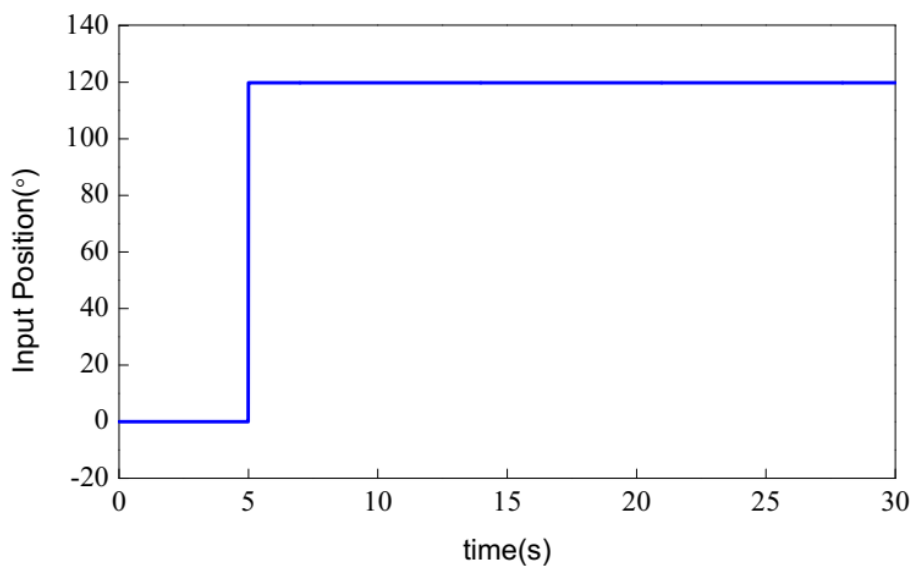

Figure 7. Desired input signal.

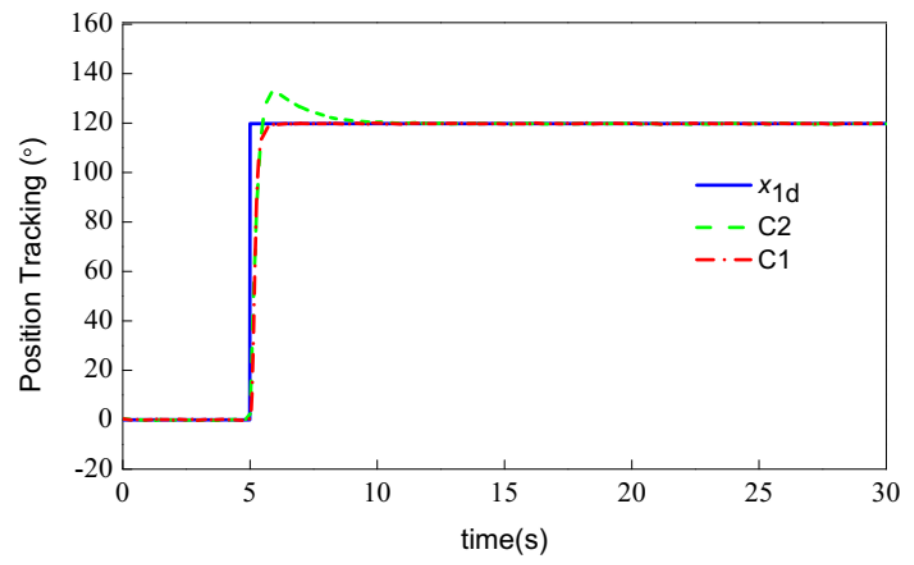

Figure 8. Position tracking performance of $\mathrm{C} 1$ and $\mathrm{C} 2$. 


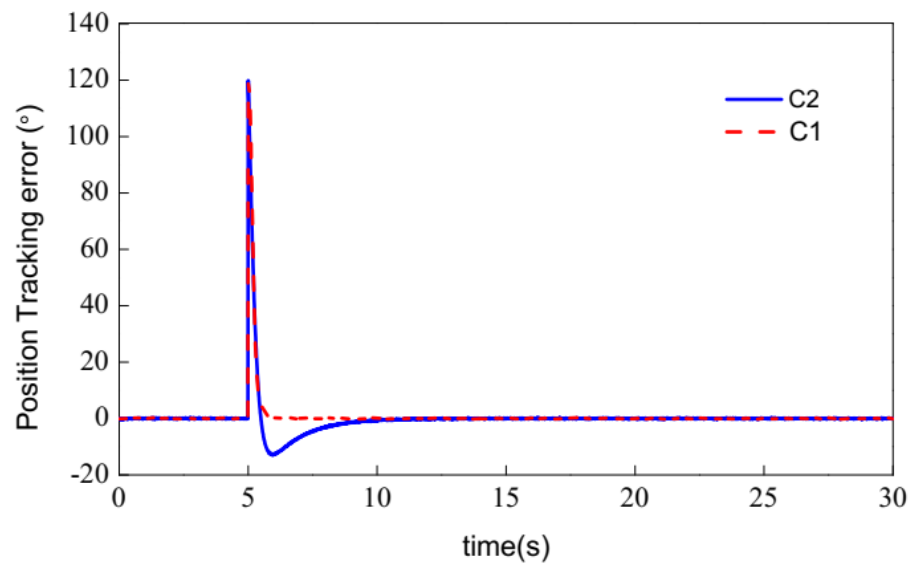

Figure 9. Position tracking error of $\mathrm{C} 1$ and $\mathrm{C} 2$.

\section{Conclusions}

In this paper, a practical controller based on the LESO and the KF was proposed for the position tracking of the robotic joint motion with variable inertia and unknown dynamics. In order to improve the ability of disturbance rejection and control precision for the robotic joint servo system, which has unknown dynamics and uncertainties, the mathematical model of the servomechanism for the robotic joint was established to derive the third-order extended state equations, which was utilized to design the LESO. The LESO was obtained with variable gain of the parameter $b$, which was related to the moment of inertia of the servomechanism. The inertia was estimated by the recursive least square method. The convergence of the estimation error for LESO showed that the position state and the total disturbance can be estimated accurately, so the model uncertainties and disturbances can be compensated effectively. TD was applied to eliminate the overshoot of the step response, while KF was used to reduce the process noise and measurement noise. Comparative experimental results demonstrated the superiority of the proposed control strategy in comparison to PID. The proposed controller had the better tracking performance in both a transient and steady-state, with time-invariant uncertainties. The limitations of the designed control law with variable gain of the parameter $b$ may cause the output to be non-smooth. Furthermore, we can choose $b_{1}$ to design the control law for the inertia values changed from minimum $J_{m}$ to median $J_{m}$ and $b_{2}$ to design the control law while inertia changed from median $J_{m}$ to maximum $J_{m}$.

Author Contributions: Conceptualization, Y.Z. and W.J.; methodology, Y.Z.; software, Y.Z.; validation, Y.Z., W.J. and X.Q.; formal analysis, Y.Z.; investigation, Y.Z.; resources, Y.Z.; data curation, Y.Z.; writing—original draft preparation, Y.Z.; writing—review and editing, Y.Z.; visualization, Y.Z.; supervision, W.J.; project administration, X.Q.; funding acquisition, Y.Z. All authors have read and agreed to the published version of the manuscript.

Funding: This research was supported by Zhejiang Provincial Natural Science Foundation of China under Grant No. LQ19E050009 and The APC was funded by Zhejiang Provincial Natural Science Foundation of China under Grant No. LQ19E050009.

Institutional Review Board Statement: Not applicable.

Informed Consent Statement: Not applicable.

Data Availability Statement: The data presented in this study are available on request from the corresponding author.

Conflicts of Interest: The authors declare no conflict of interest. 


\section{References}

1. Yang, J.; Chen, W.-H.; Li, S.; Guo, L.; Yan, Y. Disturbance/Uncertainty Estimation and Attenuation Techniques in Pmsm Drives-a Survey. IEEE Trans. Ind. Electron. 2017, 64, 3273-3285. [CrossRef]

2. Sariyildiz, E.; Ohnishi, K. Stability and robustness of disturbance-observer-based motion control systems. IEEE Trans. Ind. Electron. 2015, 62, 414-422. [CrossRef]

3. Kim, W.; Shin, D.; Chung, C. Microstepping using a disturbance observer and a variable structure controller for permanentmagnet stepper motors. IEEE Trans. Ind. Electron. 2013, 60, 2689-2699. [CrossRef]

4. Chen, J.; Patton, R.J.; Zhang, H. Design of Unknown Input Observers and Robust Fault Detection Filters. Int. J. Control 1995, 63, 85-105. [CrossRef]

5. She, J.H.; Xin, X.; Pan, Y.D. Equivalent-input-disturbance approach-Analysis and application to disturbance rejection in dual-stage feed drive control system. IEEE/ASME Trans. Mechatron. 2011, 16, 330-340. [CrossRef]

6. Shao, S.; Gao, Z.Q. On the Conditions of Exponential Stability in Active Disturbance Rejection Control Based on Singular Perturbation Analysis. Int. J. Control 2017, 90, 2085-2097. [CrossRef]

7. Garrán, P.; Garcia, G. Design of an Optimal PID Controller for a Coupled Tanks System employing ADRC. IEEE Lat. Am. Trans. 2017, 15, 189-196. [CrossRef]

8. Zhang, C.; Chen, Y. Tracking control of ball screw drives using ADRC and equivalent-error-model-based feedforward control. IEEE Trans. Ind. Electron. 2016, 63, 7682-7692. [CrossRef]

9. Wang, L.; Gao, Z.Q.; Zhou, X.S.; Han, Z.J. Exponential Stabilization of a Star-Shaped Thermoelastic Network System Based on the Extended State Observer With Time-Varying Gains. IEEE Trans. Autom. Control 2021, 66, 267-274. [CrossRef]

10. Ai, W.; Hu, L.W.; Li, X.Y. Torque ripple suppression of Switched Reluctance Motor Based on active disturbance rejection iterative learning control. Control Theory Appl. 2020, 37, 2098-2106.

11. Han, J.Q. From PID to active disturbance rejection control. IEEE Trans. Ind. Electron. 2009, 56, 900-906. [CrossRef]

12. Sun, G.; Ren, X. Neural Active Disturbance Rejection Output Control of Multi-motor Servomechanism. IEEE Trans. Control Syst. Technol. 2015, 23, 746-752.

13. Zhang, H.; Senthil, A.; Chen, F. Topology optimized multimaterial soft fingers for applications on grippers, rehabilitation, and artificial hands. IEEE/ASME Trans. Mechatron. 2019, 24, 120-131. [CrossRef]

14. Feng, N.; Shi, Q.; Wang, H.; Gong, J. A soft robotic hand: Design, analysis, sEMG control, and experiment. Int. J. Adv. Manuf. Technol. 2018, 97, 319-333. [CrossRef]

15. Chen, S.; Bai, W.; Hu, Y.; Huang, Y.; Gao, Z. On the conceptualization of total disturbance and its profound implications. Sci. China Inf. Sci. 2020, 63, 135-143. [CrossRef]

16. Wang, L.; Su, J. Trajectory Tracking of Vertical Take-Off and Landing Unmanned Aerial Vehicles Based on Disturbance Rejection Control. IEEE/CAA J. Autom. Sin. 2015, 2, 65-73.

17. Ren, L.; Mao, C.; Song, Z.; Liu, F. Study on active disturbance rejection control with actuator saturation to reduce the load of a driving chain in wind turbines. Renew. Energy 2019, 133, 268-274. [CrossRef]

18. Herbst, G. Practical Active Disturbance Rejection Control: Bumpless Transfer, Rate Limitation and Incremental Algorithm. IEEE Trans. Ind. Electron. 2016, 63, 1754-1762. [CrossRef]

19. Zhou, X.; Cui, H.; Ma, Y.; Gao, Z. The research on energy conservation controller for asynchronous motor based on ADRC. In Proceedings of the 2017 29th Chinese Control and Decision Conference (CCDC), Chongqing, China, 28-30 May 2017; pp. 4010-4014.

20. Zhang, H.; Zhao, S.; Gao, Z. An Active Disturbance Rejection Control Solution for the Two-Mass-Spring Benchmark Problem. In Proceedings of the American Control Conference (ACC), Boston, MA, USA, 6-8 July 2016; pp. 1566-1571.

21. Talole, S.E.; Kolhe, J.P.; Phadke, S.B. Extended-state-observer-based control of flexible-joint system with experimental validation IEEE Trans. Ind. Electron. 2010, 57, 1411-1419. [CrossRef]

22. Stream, L. TI Expands LineStream-Enabled Portfolio of Advanced Motion Control Solutions: C2000 TM Piccolo TM F2805x MCUs with InstaSPIN-MOTION TM. Available online: http:/ /linestream.com/news-131122/ (accessed on 1 August 2014).

23. Qing, Z.; Lin, D.Q.G.; Gao, Z.Q. On stability analysis of active disturbance rejection control for nonlinear time-varying plants with unknown dynamics. In Proceedings of the 46th IEEE Conference on Decision and Control, New Orleans, LA, USA, 12-14 December 2007; pp. 4090-4095.

24. Wang, S.; Zhu, W.; Shi, J. A High Performance Permanent Magnet Synchronous Motor Servo System Using Predictive Functional Control and Kalman Filter. J. Power Electron. 2015, 15, 1547-1558. [CrossRef]

25. Simon, D. Kalman filtering with state constraints:a survey of linear and nonlinear algorithms. IET Control Theory Appl. 2010, 4, 1303-1318. [CrossRef]

26. Shi, T.; Wang, Z.; Xia, C. Speed Measurement Error Suppression for PMSM Control System Using Self-Adaption Kalman Observer. IEEE Trans. Ind. Electron. 2015, 62, 2753-2763. [CrossRef] 